\title{
Longueurs extrémales et théorie des fonctions
}

\section{Doctoral Thesis}

Author(s):

Hersch, Joseph

Publication date:

1955

Permanent link:

https://doi.org/10.3929/ethz-a-000090221

Rights / license:

In Copyright - Non-Commercial Use Permitted 
Prom. No. 2294

\section{LONGUEURS EXTRÉMALES ET THÉORIE DES FONCTIONS}

\section{TII È S E}

PRÉSENTÉe A L'ÉCOLE POLYTECHNIQUE FÉDÉRALE, ZURICH POUR L'OBTENTION DU GRADE DE DOCTEUR ES SCIENCES MATHÉMATIQUES

PAR

\section{JOSEPH HERSCH}

DE CHENE-BOUGERIES (GENEVE)

RAPPORTEUR: M. LE PROFESSEUR DR A. PFLUGer

CORAPPORTEUR: M. IE PROFESSEUR DR M. PLANCHEREL

1955

ORELL FUSSIIARTSG RA PHIQUES S. A. Z U R I C H 


\title{
Longueurs extrémales et théorie des fonctions
}

\author{
par Joseph Hersch, Zurich
}

\section{Introduction}

Le but essentiel du présent travail est de mettre en lumière un champ d'applications de la méthode des longueurs extrémales, due notamment à Ahlfors et Beurling. Cette méthode est appliquée sous une forme modifiée présentant certains avantages. La longueur extrémale est un invariant conforme.

On peut caractériser par des longueurs extrémales les autres invariants conformes que sont le module d'un quadrilatère ou d'un domaine doublement connexe (cette propriété est connue), et, pour un domaine de Jordan, la mesure harmonique d'un arc-frontière en un point et la distance hyperbolique de deux points.

En même temps que des formules exactes, nos méthodes de variation fournissent d'utiles évaluations de théorie des fonctions: les unes précisent, parfois de façon essentielle, des inégalités connues (de Nevanlinna, Ostrowski, Sario, Strebel); les autres concernent des problèmes nouveaux.

Nous dirons toujours "courbe fermée" pour courbe de Jordan, ,arc" pour arc de Jordan et ,courbe“ pour courbe ou arc de Jordan; une ,coupure" d'un domaine sera un arc de Jordan à extrémités sur la frontière. Tous les domaines considérés seront supposés définis dans le plan complexe ou sur une surface de Riemann.

Les principaux résultats de ce travail ont été annoncés dans trois Notes aux Comptes rendus [7], [8], [12]. Une publication ultérieure [10] donnera des applications des méthodes développées ici aux fonctions pseudo-analytiques et aux transformations quasi-conformes, ainsi qu'à une classe plus générale de fonctions; la plupart de ces résultats ont été sommairement annoncés dans une autre Note aux Comptes rendus [11].

J'exprime ici ma vive reconnaissance au Prof. A. Pfluger : il m'a fourni l'essentiel de ma formation en théorie des fonctions, et c'est à lui que je dois d'avoir étudié la méthode des longueurs extrémales ; je lui sais particulièrement gré de ses conseils précieux et de sa bienveillance constante. Je remercie aussi vivement le Prof. B. Eckmann, dont je suis depuis 
longtemps l'assistant et qui m'a toujours encouragé dans mon travail; ainsi que le Prof. $M$. Plancherel, corapporteur de ma thèse, pour toute l'attention qu'il lui a consacrée. - D'autre part, on verra au Chapitre III que bien des applications m'ont été suggérées par le livre si riche de contenu ,Eindeutige analytische Funktionen" de $R$. Nevanlinna.

\section{TABLE DES MATIERES}

Introduction ................ 301

Chapitre I. La longueur extrómale

$\S$ 1. Figures et configurations. Quadrilatères et leurs modules . . 303

§ 2. La longueur extrémale d'une famille de courbes . . . . . . 303

§ 3. Longueurs extrémales et modules. Propriétés des modules . 307

Appendice du Chapitre I. Les familles numériques . . . . 311

Chapitre II. Mesure harmonique et distance hyperbolique

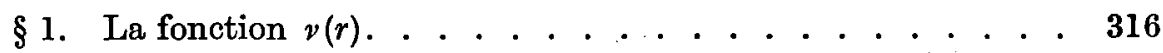

§ 2. Mesure harmonique et longueur extrémale . . . . . . . 319

§ 3. Distance hyperbolique et longueur extrémale . . . . . . 321

\section{Chapitre III. Applications}

$\S 1$. Variation de la distance hyperbolique par une déformation du domaine . . . . . . . . . . . . . . . 322

§2. Variation de la mesure harmonique par une déformation du domaine ................. 323

§3. Applications. Théorème général de Phragmén-Lindelöf . . 325

\$4. Variation d'une fonction harmonique dans des domaines simplement connexes emboîtés . . . . . . . . . . . . 329

§ 5. Evaluation par défaut de la distance hyperbolique. . . . . $\mathbf{3 3 0}$

§6. Rayon intérieur, mesure harmonique et modules . . . . . 333

§ 7. Remarque sur les théorèmes de Koebe et d'Ahlfors . . . . 335

Bibliographie . . . . . . . . . . . . . . 336 